\title{
Focus group reflections on the current and future state of cognitive assessment tools in geriatric health care
}

\author{
Jocelyne C Whitehead' \\ Sara A Gambino' \\ Jeffrey D Richter ${ }^{2}$ \\ Jennifer D Ryan ${ }^{1,3,4}$
}

'Rotman Research Institute, Baycrest, ${ }^{2}$ Independent Human Factors Consultant, Toronto, ON, Canada; ${ }^{3}$ Department of Psychology, ${ }^{4}$ Department of Psychiatry, University of Toronto, Toronto, ON, Canada

Correspondence: Jennifer D Ryan Rotman Research Institute, Baycrest, 3560 Bathurst Street, Toronto, ON M6A 2EI, Canada Email jryan@research.baycrest.org
This article was published in the following Dove Press journal:

Neuropsychiatric Disease and Treatment

12 June 2015

Number of times this article has been viewed

Objective: This study provides insight into the thoughts and opinions of geriatric health-care professionals toward cognitive assessments and the use of emerging technologies, such as eyetracking, to supplement current tools.

Methods: Two focus group sessions were conducted with nurses and physicians who routinely administer neurocognitive assessments to geriatric populations. Video recordings of the focus group sessions were transcribed and a thematic analysis was performed.

Results: Participants reported the need for assessment and diagnostic tools that are accessible and efficient, and that are capable of accommodating the rapid growth in the aging population. The prevalence of more complex ailments experienced by older adults has had repercussions in the quality of care that the clients receive, and has contributed to lengthy wait times and resource shortages. Health-care professionals stated that they are hampered by the disjointed structure of the health-care system and that they would benefit from a more efficient allocation of responsibilities made possible through tools that did not require extensive training or certification. Eyetracking-based cognitive assessments were thought to strongly complement this system, yet it was thought that difficulty would be faced in gaining the support and increased uptake by health-care professionals due to the nonintuitive relationship between eyetracking and cognition.

Conclusion: The findings suggest that health-care professionals are receptive to the use of eyetracking technology to assess for cognitive health as it would conserve resources by allowing frontline staff to administer assessments with minimal training.

Keywords: neurocognitive assessment, eyetracking, focus groups, geriatric health care

\section{Introduction}

Health-care professionals are straining to provide comprehensive care to a population that is progressively aging and rapidly expanding. ${ }^{1}$ With more complex illnesses and more frequent visits to the doctor, there is a growing concern that the influx of elderly clients requiring care is exhausting resources. ${ }^{2}$ Physicians are seeking quick and reliable screening tools to isolate the individuals who require extra attention from those who would not benefit from medical intervention. ${ }^{3}$ Signs of cognitive impairments generate a great amount of concern among the elderly population, and there is a significant need for the development of tools that are capable of a quick diagnostic screen for a diverse client population.

Currently, screening, diagnosis, and assessment of the severity of age-related cognitive disorders such as mild cognitive impairment (MCI) and Alzheimer's disease (AD) rely on paper-and-pencil or computer-based neuropsychological assessments. ${ }^{4}$ Neuropsychological assessments require extensive verbal communication between 
the physician and the client, as well as motor control by the client, but difficulties may arise in the assessment when the client is not fluent in the physician's language or presents with motor infirmities. The time required to administer an extensive neuropsychological test battery can be a lengthy process, as well as an expensive one. Administration of neuropsychological batteries requires extensive training, and the interpretation of the results must be done by a licensed physician. As such, neuropsychological batteries are timeand resource-consuming.

Moreover, there is an increasing demand for self-screening tools of cognitive function that have good sensitivity and specificity. There are currently a few screening tools used to assess cognitive health that do not require administration by a professional, that are brief and that are comparable to the reliably used Mini Mental State Examination (as reviewed by Cherbuin et al). ${ }^{5}$ Some of these include the Concord Informant Dementia Scale and the Informant Questionnaire on Cognitive Decline in the Elderly; both are extensive questionnaires that address issues related to memory, judgment, orientation, and the evaluation of other daily activities that can become significantly worse over time for individuals with cognitive impairments. ${ }^{5}$ Self-screening tools encourage consumers to become better educated in health care and participate in decision-making, but such tools are lacking in the current marketplace. Thus, a more universally applicable method of cognitive assessment is warranted: one that promotes ease of administration, does not rely on language or complex motor skills, and can be made available as a self-screening tool. The use of eyetracking technology has been proposed as a means to fill this gap. ${ }^{6}$ Research, including work from our group, has demonstrated that monitoring eye movements is an objective and convenient way to test memory, without requiring overt (verbal or motor) responses from the client. ${ }^{7}$ Where the eyes look, and for how long, is heavily influenced by what we remember. In particular, eye movement patterns can be diagnostic of the integrity of the medial temporal lobes, a region that is critically affected in $\mathrm{MCI}$ and in $\mathrm{AD} .{ }^{6}$ In healthy adults, eye movement patterns of memory correlate with medial temporal lobe activity. ${ }^{7}$ Amnesic patients, who have severe memory deficits due to lesions in the medial temporal lobes, do not show the same eye movement patterns as people who have normal memory function..$^{8-11}$ More recent work has shown that by using machine-learning techniques, measures derived from eyetracking may be used to accurately detect cognitive deficits in individuals with MCI. ${ }^{12}$ Using a preferential viewing task, Crutcher et al were able to distinguish clients with MCI from healthy controls and individuals with Parkinson's disease; again, demonstrating the usefulness of eyetracking in screening healthy versus pathological memory function. ${ }^{13}$

For years, empirical research has used measures derived from eyetracking in order to understand the changes in cognition that accompany neuropsychological, neurological, or neurodegenerative disorders. ${ }^{7}$ The translation of such findings from basic science into the development of assessment tools that can screen for, or diagnose, clinically significant cognitive decline is in its infancy and still requires more research regarding the usability and validity of any particular eyetracking-based assessment. However, the basic research that has been done to date collectively suggests that eyetracking-based assessments have the potential to fill a critical gap in health care by providing a means for quick and effective screening and diagnostics. In particular, there are advantages to using eyetracking-based assessments in multicultural or urban settings in which language fluency, education, and/or literacy levels may vary. In addition, there are advantages to using a non-verbal task with no motor requirements, except for the movement of the eyes themselves, with clients who may have language comprehension or production deficits or have restricted mobility due to stroke or other brain injuries. To determine whether eyetrackingbased assessments could augment current tools, as well as to more broadly uncover the considerations for launching new technologies and assessments in the health-care community, we consulted with health-care professionals through a focus group methodology. We engaged health-care professionals who routinely administer neurocognitive assessments to geriatric populations, to provide us with insight into the following three questions:

1. What has changed in the health-care environment over the past 10 years in the health-care system, particularly with respect to diagnostics?

2. How will technology be integrated into health-care system in the future?

3. What are the challenges in moving a tool from research to accepted diagnostics?

The focus groups were meant to generate authentic discussions and provide information that would ultimately assist in the research, design, and practical implementation of eyetracking-based assessments to screen for cognitive decline and/or diagnose specific cognitive disorders within a health-care setting. The questions were formulated with the intention of extracting relevant concerns and opinions on the broad topic of user-friendly technology in diagnostics. Ultimately, the participants in the focus groups were asked 
to provide their opinions regarding the potential future use of eyetracking-based assessments; however, the primary goal of the discussions was to understand the current state of the health-care system and its clients with particular reference to the development and use of new technologies to assist in screening and/or diagnosis of cognitive decline.

\section{Methods}

\section{Participants}

Neurologists, neuropsychologists, geriatricians, and supporting nurses were recruited from health-care facilities within the greater Toronto area. Health-care professionals were included in the study if their roles incorporated administering and/or interpreting standardized neuropsychological assessments to evaluate cognitive health. To encourage discussion and promote the honest expression of opinions, participants were placed into one of two focus groups with those who had similar responsibilities within the health-care system; one focus group comprised the nurses $(\mathrm{n}=3)$ and the second group comprised the individuals with $\mathrm{MD}$ and/or $\mathrm{PhD}$ designation (ie, neurologists, neuropsychologists, and geriatricians; $\mathrm{n}=6$ ). Participants provided written informed consent for participation in the focus group session and for audio and video recordings. Participants were each compensated with a CDN $\$ 50.00$ gift card to a local bookstore.

\section{Procedure}

Focus groups are commonly used to obtain valuable insight into the views and opinions of health-care professionals, and to assess outlooks on health services. ${ }^{14}$ Focus groups were held at Baycrest, a large geriatric health sciences center, and were guided by an independent human factors professional mediator (author Richter). The human factors professional provided activities and asked open-ended questions to allow for group dialogue and the open expression of thoughts and opinions. The professional also served to seek and provide clarification and derive consensus from participants where appropriate. ${ }^{14}$

The goal of the focus group session was to gain insight regarding the opinions of health-care professionals on the value of current neuropsychological tools and the use of emerging technologies, such as eyetracking devices, to assess cognitive health. The session consisted of a series of individual activities each followed by a facilitated group conversation. The activities were designed to minimize the effect of groupthink by allowing each individual participant to formulate his/her own view before engaging in a group conversation. Three activities were used to concentrate the group discussions on the goal. For each activity, participants were given 5-10 minutes to independently complete the task prior to the group conversation.

\section{Activity I}

What has changed in the past 10 years? (Participants complete a worksheet listing positive and negative changes around the topics of clients and the health-care system, training, and diagnostics.)

\section{Purpose}

To facilitate a discussion around which changes are seen as positive versus negative, the sources and causes of such change, and the barriers and drivers of change acceptance.

\section{Activity 2}

Draw what you think your office will look like in 50 years.

\section{Purpose}

The goal of this activity is to gain insight into how various tools and technology will be integrated into the daily events of health-care professionals, and whether these changes will be willingly received or dismissed.

\section{Activity 3}

Steps and barriers to new diagnostics. (Participants fill out a time line listing steps and considerations as well as barriers and obstacles to move a diagnostic technique from a "basic research insight" through to an "adopted diagnostic technique".)

\section{Purpose}

To uncover insights around how to create awareness, acceptance, and usage of new screening and diagnostic techniques, while surfacing concerns and considerations about how to undertake such an effort, with particular reference to eyetracking technology.

Following independent completion of each activity, the human factors professional (Richter) directed each individual to provide comments on his/her work, and allowed for unprompted input from the other participants. He promoted additional dialogue solely to clarify statements made by the participants. The duration of each focus group session was 2 hours. Discussions were videotaped and audio-recorded for later analysis.

\section{Analysis}

The recordings of the focus groups were transcribed (Gambino) and transcriptions were verified by all coauthors 
(Whitehead, Richter, Ryan). All participants and identifying information was anonymized in transcripts. A thematic analysis was conducted (Whitehead) to highlight specific themes that were expressed by the physicians, nurses, and the groups collectively. The transcripts were reviewed several times to ensure familiarity during this process. Relevant themes were noted and the transcripts were reviewed for additional themes (Ryan) and overarching concepts that represent the discussion. ${ }^{15}$ This qualitative approach allows for themes to emerge that are grounded within the data itself, and this approach had been applied in our previous research. ${ }^{16}$

\section{Results}

Specific comments from the three nurses (N1-3) and the six physicians (P1-6) were noted accordingly.

\section{Activity I}

What has changed in the past 10 years? (Participants complete a worksheet listing positive and negative changes around the topics of clients and the health-care system, training, and diagnostics.)

Focus group sessions presented several themes relating to the changes seen in the clients ("older", "complex"), the health-care system, training, and diagnostics over the past 10 years ("lack of resources", "lack of training", "value-add for technology"). They recognized that the client population was expanding to cover a broader age range, with more complex ailments. The clients were identified as being more informed and more accountable for their health, with a particular desire to seek out an earlier diagnosis and treatment. The wait lists for these services have been a growing concern in the health-care system, and the lack of medical personnel is contributing to this pressing issue. The focus group participants are discouraged by the ongoing cuts to valuable resources, and they worry that the clients are not receiving the continuity of care that they deserve. They are hopeful that as more partnerships develop within the medical community, there will be an increase in the development of high-caliber tools that can be used by individuals at any level. The current diagnostic tools being used are more sensitive, informative, and less invasive, and therefore have been received more willingly by the client population.

\section{Clients and the health-care system}

The health-care professionals described the changes occurring within the current client population:

older, more complex, but managing to be engaged longer in life in the world. (P6)
They are now required to cater to a wider age group that is more knowledgeable, including a greater number of younger clients:

... [the clients] are more willing to admit something's wrong, or their families are willing, and so they are going and asking for assessment and interventions when younger. (N3)

People are more equipped with the knowledge of health risks, and are therefore seeking earlier and more advanced diagnoses.

... [clients are] informed, more tech-savvy.... Clients to me seem younger. I've only been in business 10 years [laughs] but to me, a 70-year old now is not what a 70-year old used to be. (P5)

The health-care professionals stated that their healthy clients wanted to receive early assessments so that they can begin taking preventative measures if necessary. However, alongside this shift in health-care engagement, there remain many individuals who arrive at care facilities later in life, as they lack the support system that would have otherwise recognized the onset of impairments:

Clients arrive to us, they're quite advanced, because they may live alone for a long time, mask their impairment, because the children tend to live away. (N1)

In these advanced stages of illnesses, the health-care professionals expressed that the ability to diagnose and provide a course of treatment becomes increasingly challenging, and adds to the backlog of client care. The families of the clients have more responsibility at these stages:

... sometimes when they're coming to us they're quite advanced, they're incontinent, they don't sleep at night, they wander, and the family is stuck with them at home because there's no other solution. (N1)

With the growth of clients seeking help for cognitive health issues, there is an increase in wait times for services. The nurses expressed that wait times for consultations, tests, referrals, and lab results quickly accumulate and often have harmful repercussions:

The impact of the waitlist. So, it's a delay in diagnosis, it's putting the family through a lot more stress, having to wait a longer period of time for those results. (N3)

The wait times not only negatively impact the families, but they also may initiate stigma against the physician. Rather than putting blame on the health-care system, the 
client and their families focus their negative energy on the doctor for the delay in prognosis:

... the client perceives the doctor as incompetent when they come. (N1)

The financial state of the health-care system acts as a heavy contributor to these wait times, as extreme measures are taken to save costs:

... they keep cutting, cutting, cutting, everything, cut staff, cut programs. (N3)

The health-care professionals worried that with these cutbacks, the clients do not receive the personalized longterm care they deserve:

... not enough individualized care, not much time for indi-

vidualized care .... (N2)

Both the physicians and nurses reported that the current health-care system is heavily task-oriented, and that there is little constancy with treating clients:

... the person doesn't really have the continuity of care .... (P2)

The physicians acknowledged that there has been a tendency in clients to want excessive testing:

... I get a lot of demands for treatments that don't exist or to repeat or extend a lot of technology-based tests, which are not only not productive but counter-productive. (P2)

Clients are not receiving the reassurance that they need from current cognitive assessments, and are seeking out more advanced tools with a consumer mentality:

... [there is] a concept of entitlement and consumerism. (P2)

... people coming in nowadays saying, 'I want'. (P6)

One physician noted that, to date, new technologies have not contributed to health care in a way that is most needed:

... what is very interesting is that despite the proliferation of this technology, waiting lists don't seem to shrink very much. (P2)

\section{Training}

There were differences in opinions expressed by the nurses and physicians with respect to the evolution of training over the years. The physicians were overwhelmed by the lack of training that medical professionals are receiving in the field of geriatrics:
We all grumble that there's still no mandatory training in geriatrics in undergraduate schools, (P1)

while the nurses were seeing more opportunities for individuals in their field. The physicians identified that part of the medical training must focus on eliminating predetermined judgments regarding elder clients:

There's an ongoing negative perspective that biases against seniors in our health care system that impacts on our trainees.... (P1)

However, despite this purported negative bias, they are observing an influx of trainees into the field of geriatrics:

... there's really a ground swell interest in geriatrics and therapy from the trainees. (P5)

The participants hoped to see not only more trainees moving into careers in geriatrics, but also to see more teamwork and collaboration developing within the field:

Most of us will be working with teams, certainly those of us in geriatrics are working with teams now. But the teams are gonna be, the inter-professional team. (P1)

The nurses expressed the advantages in collaborative practice in client long-term care:

... [training] has improved due to more collaborative practice between the different disciplines. And I think that's really nice, you know, where the physicians and allied health, and nurses are working together, sharing together.... (N3)

The nurses hoped to see more connections made between research and health-care personnel:

... all the research and bedside care is disjointed.... (N1)

One of the nurses recognized the value in connecting research to care after witnessing another health-care facility enact this strategy:

... they're so connected with each other that I was really jealous seeing what researchers give back to the bed side and vice versa, and that's something we could implement. (N1)

\section{Diagnostics}

Physicians and nurses were in agreement that clients benefit from the use of new technologies to attain an earlier diagnosis:

an earlier start to treatment. Well, you buy six months. That's better. (P5) 
... there's no question, they're more sophisticated, you get a lot more information more readily, give or take a little for most of the low-risk environment.... (P6)

Additionally, a nurse emphasized the advantages new diagnostics have for the well-being of the client during the assessment:

... right now they're looking at picking up Alzheimer's at a very early stage by looking at the eye ... so I think that diagnostically it will be perhaps less invasive. (N3)

Advancements in diagnostics were also thought to improve the quality of the results:

... there are two things that I've seen. One is improved quality. So, we've moved from, for example, low-field strength MRI to high-field strength MRI, [...] with some improvement in the diagnostic accuracy. (P2)

As noted earlier, although new diagnostic tools bring some advantages, concerns still surround the time needed to obtain such tests:

the negative: waitlists for these tests.... (N3)

Although the majority of the discussion around the evolution of diagnostics was optimistic, there was a critical concern expressed by both the physicians and nurses. Namely, that although there is increasing ability to provide an early diagnosis, they collectively feel there is little value in it:

Now one of the big, big criticisms of this ... is to what end? Because we know the implications of early diagnosis, even if it were possible, really, we're not sure what the end is, when we have very little to go with to tell people, "You've got a bad future. And don't try to get life insurance anymore because you've just lost it". (P6)

The physicians noted that although there is ongoing research regarding drug treatments for dementia, currently they do not have effective treatments for individuals who have been diagnosed. Thus, there is debate in the health-care community regarding whether there is merit in telling the client of the status of their chronic, incurable illness.

\section{Activity 2}

Draw what you think your office will look like in 50 years.

The themes that emerged in the second activity revolved around "technology", "care", and "efficiencies". Health-care providers require reliable diagnostic tools to assist them in making accurate prognoses. ${ }^{4}$ Participants were optimistic about a future of more advanced and efficient technology incorporated into their offices and daily practice and were in agreement that the use of medical devices to aid diagnoses and provide care should be supported, and:

they're sound proof ... it would increase your productivity and reliability. (P5)

The concepts of accessible diagnostics and telemedicine were predicted as having a large presence in the future of health care:

... there's a lot of positive to technology. I think you can bring people together from different areas.... (N2)

The participants were passionate about the prospect of connecting with experts around the world, and having the ability to treat clients globally:

... you'd want a big giant screen for telemedicine. I mean, we should be doing that now. But, we'll probably be doing a lot of this, people will be in their homes, people will be in different parts of the country or province or city or whatever. (P5)

The health professionals had a largely uniform conception of how they envisioned their "offices of the future". There was a general emphasis on the increased use of computers and touch-screen monitors:

I did change my usual testing table where I used to do paper and pencil for people, [the table] can now have a computer on it because I think we'll do more and more computerbased testing. (P3)

The professionals also imagined more office space allocated to comfortable sitting areas, so as to encourage a more relaxed environment:

Two armchairs for people for more intimate discussions with the client. (N1)

Although these types of tools were identified as essential for treating and managing the growing population of geriatric clients, there was the fear that the increase in technology would result in the loss of valuable person-to-person contact needed for successful healing. They reported that personal contact was an aspect of health care that they hoped to persevere well into the future:

I still believe that the essence of what we do is based on our communication and touch. Real touch. Touching is emotional touch and physical touch. (P6) 
The proposed solution to maintain personal contact in the face of anticipated increases in technology use was a reallocation of resources, so that the precedence remains in the "care" aspect of health care. One of the physicians reported that valuable time is currently being wasted on mundane tasks:

there's a lot of time in here that we spend on basic things like, right now we have paper and pencil tests, so we have to score them. (P4)

Advanced diagnostic tools could save time, reduce expenses, and realign the focus on the clients' individualized needs. The theme of time conservation frequently resurfaced throughout the focus groups, as several physicians emphasized that there must be time allocated for contact between the healer and the sick, as it is important for developing trust and delivering the proper subjective care for each client:

... I hope that there's always going to be someone at the end of the day who can do face to face time if needed. (P3)

Participants were in agreement that future diagnostic and screening tools that could be administered by frontline staff would not only maximize time for physicians to deliver care, but it would also reduce costs:

... it's not cost effective to have a psychiatrist, like myself, spend two hours or bill the government for a certain amount of time to do cognitive testing when someone who, someone else just as skilled can do that, for less. (P5)

Training frontline staff directly was suggested as a more effective strategy in implementing new practices, in replacement of information being conveyed through middle managers:

... it does it a disservice to the frontline, the allied health, even MDs, by giving it to your middle managers, who aren't that dynamic.... (N2)

Physicians and nurses alike envisioned the development of diagnostic or screening tools that could be administered by nurses, which would allow other tasks necessitating expertise to be reserved for the physicians:

... nurses will start doing more diagnostic, and have allied teams, not just nurses. I think that the current medical hierarchy can't sustain itself indefinitely. (N2)

A physician in the group extended this prediction by eliminating the need for qualified personnel to administer the tests altogether:
... have a robot or a computer test them ... it's not a good resource of a person's time. (P5)

The urgency of reforming diagnostics became prevalent as participants discussed the growing cohort of older adults and the increased complexity of health problems that present with increasing age. Participants felt that more resources (ie, money and personnel) will be needed to diagnose and treat individuals who present with more extensive health concerns. The nurses articulated their apprehension that an excessive client load will cause the quality of care provided to be reduced:

... it's all about processing more people, moving things faster, getting more people in, working with what you have, but pushing.... (N2)

and

... things are being pulled away from the person to person contact. It's all about efficiency and people cost money. (N2)

They were hopeful about the restructuring of the health-care system in the future:

... something is going to happen to make it a more efficient sys-

tem. With the same money, but in a different thinking. (N1)

For the nurses and physicians, increasing the use of technology could improve work efficiency that would then allow for a focus on client care. They further noted that current tools were not adequately administered and that the use of training programs would increase the reliability of the results:

... one of the things for primary care physicians is that very

few have actually been trained on how to do cognitive testing in the first place. So people may take a form, and ask the questions that are on the form, but may not know the, you know, may not know how to do it properly, or they may change it around ... so having it standardized in a way would a) would make more of them occur probably because if it was more accessible, it can be more standardized. (P1)

There was a general consensus that the future of health care would benefit from the use of technology to improve efficiency and reserve time for person-to-person care. Specifically, technology that would create simple, accessible, and standardized diagnostic tools that could be administered by less qualified personnel would allow for physicians to conserve their time and focus more on providing individualized care for the client. These types of tools were further seen as a way to encourage collaboration among physicians, nurses, and allied health personnel, as well as between care providers 
and researchers. The physicians and nurses further noted that standardized diagnostic and screening tools through the use of advanced technologies could support the expansion of telemedicine, allowing for individuals across the world to receive equal access to health care.

\section{Activity 3}

Steps and barriers to new diagnostics. (Participants fill out a time line listing steps and considerations as well as barriers and obstacles to move a diagnostic technique from a "basic research insight" through to an "adopted diagnostic technique".)

Themes that emerged from this final activity centered on "rigorous testing", "inclusion of frontline staff", and "wait time reduction". The participants consistently recommended that extensive research must be done on the reliability and accuracy of potential diagnostic tools before approaching funding sources and investors. The participants recommended that multisite testing would be of value to achieve standardization and reliability of the tool:

... [use] different groups and different subjects, and I guess that's why I thought about multi-sites, you know, because it's a pooling of resources, it's a pooling of information and a pooling of results ... that you can have to use this, to evaluate it. (N3)

However, attaining grant funding to support such research on new diagnostic tools was consistently anticipated as the greatest hurdle:

So that's the barrier, it's that granting is just seen as, recently it's changed how they give grants. Recently it's hard to get CIHR (Canadian Institutes of Health Research) grants nowadays, it's very competitive. (P5)

The nurses in the focus group sessions suggested that acceptance of a new diagnostic tool would benefit from a steady correspondence with the frontline staff who would be using the tool to gauge their interest and promote education:

you have to get your people on the frontline to really want to do it. (N2)

The physicians noted they would need to be convinced that the tool would be more beneficial than any other on the market:

... all the other ones are supposed to be reliable, universally marketable, $[\ldots]$ the question you said was, "what will motivate a doctor to use it?". (P6)
There was also acknowledgment that many physicians may not be receptive to implementing new technologies:

... not all doctors are ready to explore new tools, and it's difficult, many doctors are older, and they're set in their ways.... (N1)

The greatest hurdle that was consistently reported by both the nurses and the physicians in the development of a successful diagnostic tool was wait times. In order to gain support and funding, they suggested that any new tool would need to provide a quick diagnosis, while reducing wait times:

Think about all the things that we just talked about here, the scans, the assessments, the waiting for this one, then the neuropsychologist isn't ready.... (N2)

The nurses noted that clients may not have the emotional stamina to endure the constant anticipation of test results:

... it's really putting them through an emotional roller coaster that could be eliminated if these tests were just done quicker. (N2)

The nurses further commented that shorter wait times would ultimately benefit the caregivers and greatly reduce stress:

... all this diagnosis, somebody needs to take that person to the doctor for referral, then somebody needs to take them to that MRI, then somebody needs to take, with all the results, back to the neurologist, and that all falls on the caregiver.... (N1)

Diagnostic tools that are simplistic in their output could successfully reduce wait time and reduce the stress that is felt by the physicians who aim to provide care and treatment plans in a timely fashion:

... doctors go through lots of frustrations. By the time the client comes, the MRI is not ready. The testing didn't come.

The x-ray is not ready yet. You know, by the time they're trying to diagnose, they have their own frustrations. (N1)

The physicians identified "automated" and "reliable" as two key characteristics that any new diagnostic tool must have to achieve acceptance by the medical community.

My personal view is that automated methods are more able to extract data in a reliable fashion. (P2)

Through automation and reliability, the physicians noted that tools could help eliminate risks of misdiagnosis and elevate confidence in their diagnoses. 
... when it comes from cognitive testing, from my point of view, we need something that is accurate, which is available, which does not depend on having a very small cadre of highly trained individuals and is interpretable by primary care physicians and neuro-physicians; (P2)

... for those of us who aren't good at neurological exams anymore, that's me, might jump straight to a more sophisticated test. Sometimes you might not need to do that if there's something easier and cheaper and just as valid and reliable; (P5)

If this test could somehow make it, give the family physicians more confidence to make that diagnosis. And identify the folks where, you know, based on the results of this [test], you really need to get into a specialized clinic.... (P1)

The physicians emphasized that the value of any new tool also rests in its ability to be applied to a diverse population:

... the problem that I have with all cognitive testing, that I have a group of testing, that do not speak English. I have patients who are not literate, I have patients who cannot read an eye-chart, cannot read numbers on an eye-chart. So, for my point of view, the development of a test that does not depend on literacy level, that does not depend on linguistic abilities, is not culturally bound, all of those things are extremely important. (P2)

When specifically asked about the possibility of using eyetracking as a tool to screen for early cognitive decline, there was a general support for the development of eyetracking-based assessments:

... this whole idea of using eye-movement as a way to access cognitive process or to investigate cognitive processes I thought was very interesting...; (P2)

I'd have it installed in my office in 2 hours. (N3)

... family doctors would like to give them quick and easy tools to do this in the office instead of making the patient wait for a long time, dement further .... (P5)

In particular, there was consensus that eyetracking may be a tool that would bypass testing challenges related to language use or literacy levels:

... I think something that would involve eye movements might circumvent some of the problems associated with those particular issues that I've raised. (P2)

However, not all initial responses were positive:

That would be really stupid. Eye-tracking. Like your eye?. (P1)
These varied responses accentuate the value of using accurate and informative marketing for such a product, as with further conversation among the professionals, the physician who initially expressed a negative opinion was ultimately more receptive of the concept:

Cool. Okay. (P1)

\section{Discussion}

The discourse during the focus group sessions identified themes and provided valuable insight into the current state of client care as well as the functionality of the assessment tools used within the health-care system. In particular, the professionals primarily expressed their frustration with the inadequate level of care being provided to the client population. The frustrations stemmed from client anxiety, attributable to the inability of the health-care system to accommodate the changing population. The professionals suggested that the structure of the health-care system is damaging to its functionality and readily contributes to the backlog of clients, the lengthy wait lists, and ultimately the inability to provide high caliber care. These concerns are accentuated as the client population is reportedly more demanding in receiving suitable care.

The physicians recognized that there has been a growing phenomenon around health care being regarded as a commodity. The clients are more informed and conscientious of the health issues that can arise with old age, and therefore eager to receive an early diagnosis and take the necessary preventative measures to avoid illness. Individuals are demonstrating entitlement and consumerist behaviors to receiving the most advanced and extensive diagnostics and treatment. Health-care professionals are struggling to mediate these challenges with the limited resources that are provided, given the increasing cutbacks to funding and personnel. Physicians and nurses alike hoped to see the implementation of tools that could maximize resources, preserving time and expenses, while reducing wait times and providing the client with an early and informative diagnosis.

The health-care professionals believed that for the health-care system to function efficiently, restructuring would be required, both in terms of the kinds of screening and diagnostic tests that are conducted, and in terms of the manner of training and team development. With respect to current diagnostic tools, paper-and-pencil neuropsychological assessments were quickly recognized as a recurring source of angst for both the physician and client. Time spent completing the assessments, analyzing the results, and following up with the client was regarded as resource-intensive 
and inefficient. A quick and proficient screening tool would allow physicians to diagnose a larger number of clients, and permit more energy to be focused on the individuals that require more extensive care. Accessible and user-friendly tools that also serve as self-screening tools would satisfy a client's need for involvement and reduce strain on the health-care system. The professionals envisioned tools that would extend beyond the confines of the doctor's office to include use in commercial buildings, at home, or even via a smartphone on the go.

However, the main objective noted by the health-care professionals was to allocate more time to personalized patient care, and to develop an extensive network of specialized professionals that could provide comprehensive care. They acknowledged that the health-care community would only be receptive to novel technologies that support screening and diagnostics if those tools could ultimately aid in the preservation of time allocated for care, or could increase the functionality or efficiency of the health-care teams.

The health-care professionals acknowledged several obstacles that could prevent the implementation of new screening or diagnostic technologies into the health-care system. They suggested that the greatest challenges would be in gaining support from the health-care community and obtaining sufficient financial capital. Physicians may not be receptive to using new technology, as they have acclimatized to their own tools and practices. To convince all parties, any new product must be a cost-effective solution with minimal up-front investment for which research has shown better sensitivity/specificity than the current paper-and-pencil and other standardized computer tasks.

Although more automated screening and diagnostic tools were appealing to the participants, they were concerned that any such assessments would not address the issues that currently arise with traditional neurocognitive assessment. The health-care professionals noted that standard assessments are often unable to accommodate individuals with language difficulties, individuals who are fluent in a language distinct from that of their health-care provider, and those individuals who have a low level of education. The concern was also expressed that individuals with severe mobility issues or those who lack family support may be incapable of making routine visits to their family physician for an assessment. A more automated screening or diagnostic tool would be less likely to be adopted if these issues are not addressed. Marketing any new screening or diagnostic technology appropriately to health-care professionals would be critical for widespread implementation. As an example, eyetracking-based assessments were not initially received positively by all health professionals, yet after an open discussion of how assessments based on measures derived from eyetracking could address many of the concerns outlined earlier, the group became increasingly supportive. Eyetracking-based assessments could be administered by an assistant prior to consultation with the physician, and potentially eliminate the need for a follow-up appointment, or serve to prioritize clients. Since eyetrackingbased assessments could require minimal training, physicians could delegate the initial screening of clients to frontline staff, thereby freeing more time for client follow-up, interaction, and care that is relevant for a given diagnosis. Eyetrackingbased assessments would also alleviate financial tension by prohibiting expensive specialists from billing for the administration of such tasks. With the increasing number of older clients requiring care, medical expenses have become a significant stressor for the health-care system, and there is concern that additional funds will need to be rerouted from other initiatives to support health care. ${ }^{17}$ New screening and diagnostic technologies are more likely to gain support and widespread adoption if they can reduce overall expenses, as highlighted by the health professionals.

The growing interest in the use of eyetracking has encouraged the development of varied assessments that could be useful as a screen or a diagnostic tool for distinct neurodegenerative disorders, such as AD and Parkinson's disease. For instance, Cameron et al used eyetracking technology to illustrate that individuals with PD present impairments in their ability to make motivated antisaccades, and work is underway to develop standardized protocols that could use the antisaccade eye movement paradigm to screen for clinically significant conditions. ${ }^{18,19}$ Similarly, a recent review highlighted the use of eyetracking-based assessments to detect cognitive impairments indicative of MCI or AD. ${ }^{6}$ As each neurodegenerative disorder has unique neural, cognitive, and behavioral characteristics, screening and diagnostic tools will ideally reflect these variances with specificity and sensitivity. The use of measures as derived from eyetracking has already been shown to be a powerful tool by which to index numerous cognitive processes (eg, memory, attention, decision-making, and language comprehension). 7,20 Eyetracking-based assessments do not necessarily have to rely on language capabilities, and they have the capacity to accommodate individuals with physical impairments, which were main concerns that were expressed by the health-care professionals in the focus group sessions. Eye movement measures may be less affected by education levels or cultural influences, in contrast to other neuropsychological assessments. ${ }^{13}$ 
Eyetracking-based assessments have the ability to collect a range of information from a single screening that can then be easily interpreted and accumulated to form an expansive database of results. ${ }^{21}$ The physician could then immediately receive results succeeding test administration and follow up accordingly. Eyetracking-based assessments are well matched to current tools in telemedicine, as low- and middle-income countries require affordable, portable, and easy to operate diagnostic tools. Past research has identified that optical devices are some of the most effective tools that best match these requirements for diagnostics in developing countries. $^{22}$

It should be noted that much research remains to investigate the trajectory of eye movement markers relative to the trajectory of other cognitive neural biomarkers indicative of disease onset and progression. While basic empirical research has suggested that there is utility in adapting eye-movementbased assessments of cognitive function from lab-based paradigms into clinical screening or diagnostic tools, much work remains regarding the standardization, reliability, sensitivity, and specificity of any given eyetracking-based assessment.

Moreover, there was concern expressed by both focus groups, about the value in using any technology to provide an early diagnosis for dementia. Pharmacological treatments may have their greatest efficiency when administered prior to significant disease progression. Non-pharmacological treatments, such as psychosocial and psychological approaches, could provide benefit to individuals diagnosed at early stages with dementia, ${ }^{23}$ and there are indications that cognitive training approaches may have protective properties and provide compensatory strategies. ${ }^{24}$ The ultimate goal of using technologies to develop new cognitive assessments is to provide early screening and diagnoses. The consequences of an early diagnosis continues to be a controversial area of discussion in the health-care community, but such issues should be addressed as the advancements in technology are quickly providing us with better opportunities for developing highly informative tools. At the very least, better screening and diagnostic tools would allow clients to gain a better understanding of the disease and mentally prepare alongside their families to avoid potential harm that can accompany the illness, whether physical or mental. ${ }^{25}$

\section{Conclusion}

The focus group sessions provided a better understanding of the changing client demographics and the challenges within the health-care system that warrant a change in the nature of the screening and diagnostic tools. The small sample size of health-care professionals may limit the generalizability of our findings; however, opinions were largely similar across the two professional disciplines. The collective felt that with the aging population, there needs to be new cognitive assessments that are reliable across a population that is variable with respect to language experience, education, and mobility. The age of technology has allowed for a greater access to information, diagnoses, and treatments. Individuals are seeking to have more ownership over their health. Eyetracking-based assessments would provide opportunities to have efficient screens of health status, and could ultimately be developed as selfscreening tools. Alongside current research regarding the efficacy of pharmacological and non-pharmacological therapies for dementia, we are at the optimal time to develop efficient screening and diagnosis tools (either based in eyetracking technology or otherwise) that can accommodate a diverse client population, be delivered by frontline staff, and deliver early indicators of clinically significant cognitive decline.

\section{Acknowledgments}

This research was supported by funding from the Canada Research Chairs Program and Ontario Centres of Excellence awarded to JD Ryan. We would like to thank Alain Monnard for facilitating the organization of the focus group sessions and Tiffany Chow for helpful discussions.

\section{Disclosure}

JD Richter received payment as an independent contractor for the design and facilitation of the focus groups and thematic analysis plan. The other authors report no conflicts of interest in this work.

\section{References}

1. Statistics Canada. Annual Demographic Estimates: Canada, Provinces and Territories. Statistics Canada Catalogue no. 91-215-X, no. 2. Ottawa, ON: Statistics Canada; 2014.

2. Canadian Institute for Health Information (CIHI). Health Care Use at the End of Life in Western Canada. Ottawa, ON: CIHI; 2007.

3. Nasreddine ZS, Phillips NA, Bédirian V, et al. The Montreal Cognitive Assessment, MoCA: a brief screening tool for mild cognitive impairment. J Am Geriatr Soc. 2005;53(4):695-699.

4. Woodford HJ, George J. Cognitive assessment in the elderly: a review of clinical methods. Q J Med. 2007;100(8):469-484.

5. Cherbuin N, Anstey KJ, Lipnicki DM. Screening for dementia: a review of self- and informant-assessment instruments. Int Psychogeriatr. 2008; 20(3):431-458.

6. Pereira MLGF, Camargo MVZA, Aprahamian I, Forlenza OV. Eye movement analysis and cognitive processing: detecting indicators of conversion to Alzheimer's disease. Neuropsychiatr Dis Treat. 2014; 10:1273-1285.

7. Hannula DE, Althoff RR, Warren DE, Riggs L, Cohen NJ, Ryan JD. Worth a glance: using eye movements to investigate the cognitive neuroscience of memory. Front Hum Neurosci. 2010;4(166):1-16. 
8. Ryan JD, Althoff RR, Whitlow S, Cohen NJ. Amnesia is a deficit in relational memory. Psychol Sci. 2000;11(6):454-461.

9. Ryan JD, Cohen NJ. Processing and short-term retention of relational information in amnesia. Neuropsychologia. 2004;42(4):497-511.

10. Ryan JD, Cohen NJ. The nature of change detection and online representation of scenes. J Exp Psychol Hum Percept Perform. 2004; 30(5):988-1015.

11. Olsen RK, Lee Y, Kube J, et al. The role of relational binding in item memory: evidence from face recognition in a case of developmental amnesia. J Neurosci. 2015;35(13):5342-5350.

12. Lagun D, Manzanares C, Zola SM, Buffalo EA, Agichtein E. Detecting cognitive impairment by eye movement analysis using automatic classification algorithms. J Neurosci Meth. 2011;201:196-203.

13. Crutcher MD, Calhoun-Hany R, Manzanares CM, Lah JJ, Levely AI, Zola SM. Eyetracking during a visual paired comparison task as a predictor of early dementia. Am J Alzheimers Dis Other Demen. 2009; 24(3):258-266.

14. Kitzinger J. Introducing focus groups. Br Med J. 1995;311(7000): 299-302.

15. Braun V, Clarke V. Using thematic analysis in psychology. Qual Res Psychol. 2006;3(2):77-101.

16. McEwen SE, Polatajko HJ, Davis JA, Huijbregts M, Ryan JD. 'There's a real plan here, and I am responsible for that plan': Participant experiences with novel cognitive-based treatment approach for adults living with chronic stroke. Disabil Rehabil. 2010;32(7):540-550.
17. Canadian Institute for Health Information (CIHI). Health Care in Canada, 2011: A Focus on Seniors and Aging. Ottawa, ON: CIHI; 2011.

18. Cameron IGM, Brien DC, Links K, et al. Changes to saccade behaviour's in Parkinson's disease following dancing and observation of dancing. Front Neurol. 2013;4(22):1-10.

19. Antoniades C, Ettinger U, Gaymard B, et al. An internationally standardized antisaccade protocol. Vis Res. 2013;84:1-5.

20. Mele ML, Federici S. Gaze and eye-tracking solutions for psychological research. Cogn Process. 2012;12(Suppl 1):S261-S265.

21. Gualtieri CT. Computerized neurocognitive testing and its potential for modern psychiatry. Psychiatry (Edgmont). 2004;1(2):29-36.

22. Balsam J, Ossandon M, Bruck HA, Lubensky I, Rasooly A. Low-cost technologies for medical diagnostics in low-resources settings. Exp Opin Med Diagnost. 2013;7(3):243-255.

23. Prince M, Bryce R, Ferri C. World Alzheimer Report 2011: The Benefits of Early Diagnosis and Intervention. London: Alzheimer's Disease International (ADI); 2011.

24. Sitzer DI, Twamley EW, Jeste DV. Cognitive training in Alzheimer's disease: a meta-analysis of the literature. Acta Psychiatr Scand. 2006;114:75-90.

25. Leifer BP. Early diagnosis of Alzheimer's disease: clinical and economic benefits. J Am Geriatr Soc. 2003;51(5 Suppl Dementia):S281-S288.
Neuropsychiatric Disease and Treatment

\section{Publish your work in this journal}

Neuropsychiatric Disease and Treatment is an international, peerreviewed journal of clinical therapeutics and pharmacology focusing on concise rapid reporting of clinical or pre-clinical studies on a range of neuropsychiatric and neurological disorders. This journal is indexed on PubMed Central, the 'PsycINFO' database and CAS,

\section{Dovepress}

and is the official journal of The International Neuropsychiatric Association (INA). The manuscript management system is completely online and includes a very quick and fair peer-review system, which is all easy to use. Visit http://www.dovepress.com/testimonials.php to read real quotes from published authors. 